\title{
A NEW APPROACH FOR MICROCALCIFICATION ENHANCEMENT IN DIGITAL BREAST TOMOSYNTHESIS RECONSTRUCTION
}

\author{
Maissa Sghaier ${ }^{\star \dagger}$, Emilie Chouzenoux ${ }^{\star *}$, Giovanni Palma ${ }^{\dagger}$,Jean-Christophe Pesquet ${ }^{\star}$, and Serge Muller ${ }^{\dagger}$ \\ $\star$ CVN, CentraleSupélec, Inria, Univ. Paris Saclay, France \\ * LIGM, Univ. Paris Est Marne-la-Vallée, France \\ $\dagger$ GE Healthcare, Buc, France
}

\begin{abstract}
We propose a novel approach aimed to improve the detectability of microcalcifications in Digital Breast Tomosynthesis (DBT) volumes. Hence, our contribution is twofold. First, we formulate the clinical task through a detectability function based on an approach inspired from mathematical model observers. Second, we integrate this new developed clinical-task term in a cost function which is minimized for $3 \mathrm{D}$ reconstruction of DBT volumes. Experimental results carried out on both phantom and real clinical data show that the proposed clinical term allows the visibility of microcalcifications to be significantly improved, while preserving an overall high quality of the fully reconstructed volume.
\end{abstract}

Index Terms - Digital Breast Tomosynthesis, Regularization, Detectability, Microcalcifications Enhancement, 3D Image Reconstruction.

\section{INTRODUCTION}

Standard Mammography (MX) is the most widely used imaging tool for breast cancer screening and diagnosis. As a 2D projection imagery technique, it is affected by geometric limitations such as the superimposition of tissues which can reduce the visibility of lesions present in the breast or even generate false structures that can mislead the radiological interpretations.

In order to alleviate these limitations, Digital Breast Tomosynthesis (DBT), which is a three-dimensional imaging modality, has been proposed as an alternative technique. It relies on the acquisition of a set of $2 \mathrm{D}$ projections on a limited angular aperture. The reconstruction of a 3D volume of the imaged object from the set of acquired projections, allows the superimposition of the structures to be reduced and thus improves the visibility and the identification of the potentially present lesions in the breast. Nevertheless, DBT remains characterized by anisotropic spatial resolution, with high resolution in the planes parallel to the detector and much lower resolution in the perpendicular direction. DBT image reconstruction is considered as an ill-posed problem where iterative algorithms demonstrated superiority over one-pass algorithms such as filtered-back projection [1]. The main advantage of such methods is their ability of incorporating regularization functions that aim at alleviating the missing information issue [2],[3],[4],[5],[6]. Even though existing regularized approaches seem to provide a certain trade-off in terms of image quality, they do not account for the final usage of images by the radiologist. More precisely, the different clinical tasks of the radiologist are not formally taken into account when reconstructing images. A major challenge thus lies in the definition of regularization terms fostering a real benefit in terms of clinical practice. In DBT imaging, among the most important clinical tasks is lesion detection. In the literature, mathematical model observers were developed to assess the image quality for such specific clinical task [7]. Two main types of model observers can be distinguished, namely ideal observers (IOs) which assume a full knowledge of the statistics of the image [8] and anthropomorphic observers that mimic the human observer for a specific task [9],[10]. The latter ones provide as an output a scalar decision variable that quantifies how detectable is the lesion in a given image. In this context, our contribution is to propose a new approach for combining the task-based assessment strategy used in anthropomorphic model observers and regularized iterative DBT reconstruction algorithms. A new clinical-task based a priori term is formulated, and integrated in the reconstruction algorithm. The proposed method aims at maximizing the detectability of lesions in predefined regions of interest.

The paper is organized as follows: Section 2 formulates the reconstruction problem and describes regularized-based reconstruction methods. In Section 3, we present our main contribution, that is the introduction of a new regularization term for lesion detection enhancement. Section 4 discusses the practical implementation of the proposed approach. Finally, experimental results illustrating the benefits and limitations of our approach are presented in Section 5

\section{PENALIZED RECONSTRUCTION APPROACH}

In DBT, the physical acquisition process [11] can be simplified so that the link between the observed data to the object 
being imaged is translated into a linear model:

$$
p=A d+\epsilon
$$

where $p \in \mathbb{R}^{n}$ is a vector derived from the acquired images, $d \in \mathbb{R}^{m}$ a vector representing the unknown volume, $A \in \mathbb{R}^{n \times m}$ denotes the geometry of the system and $\epsilon \in \mathbb{R}^{n}$ is the noise. The aim is then to reconstruct an estimate of $d$ from the projections $p$. This is a challenging task because of the ill-conditioning of the projection matrix, of the presence of noise, and of the very large number of variables. An efficient way for solving this problem is to use an optimization approach where a penalized least squares cost function is minimized:

$$
\underset{d \in \mathbb{R}_{+}^{m}}{\operatorname{minimize}} \frac{1}{2}\|p-A d\|_{2}^{2}+\Phi(d) .
$$

Hereabove, $\Phi$ is a regularization function introduced to encourage the solution to lie in a specific desirable space, thus improving the robustness to the noise. Several choices have been proposed for function $\Phi$, taking into account range constraints, spatial regularity, or sparsity in a possibly transformed domain. Although the addition of such regularizers led to interesting results in terms of overall image quality, it is worth emphasizing that, to the best of our knowledge, none of them take into account the clinical finality of the scan exam, that is determining and localizing the presence of clinically relevant patterns in the observed tissues. In the particular case of DBT, one of the performed clinical tasks is the detection of calcifications, taking the form of bright and very small objects with respect to the overall volume. This makes the detection task very challenging, and thus raises the need for new regularization terms that not only integrate global regularity priors on the volume, but also aim at enhancing the visibility of the lesions.

Therefore, we propose to integrate in the cost function (2) a new penalty term that corresponds to the maximization of some detectability criterion in the reconstructed volume $d$. The optimization problem now takes the form:

$$
\underset{d \in \mathbb{R}_{+}^{m}}{\operatorname{minimize}} \frac{1}{2}\|p-A d\|_{2}^{2}+\Phi(d)-\alpha D(d)
$$

where $D: \mathbb{R}^{m} \rightarrow \mathbb{R}$ denotes the detectability function, with weight $\alpha>0$, that will be built for the purpose of enhancing the visibility of calcifications for the radiologist in some predefined regions of interest.

\section{PROPOSED DETECTABILITY FUNCTION}

Let us consider a set of $q$ vectors $\left(r^{i}\right)_{1 \leq i \leq q}$ of intensity values in regions of interest where, for every $i \in\{1, \ldots, q\}, r^{i} \in \mathbb{R}^{k}$ $(k \leq m)$, is the vector delimiting a zone where a calcification is potentially present. For every $i \in\{1, \ldots, q\}$, we introduce the decimation operator $S_{i}$ that extracts a region of interest $r^{i}$ from a given volume $d$ as follows:

$$
\begin{aligned}
S_{i}: & \mathbb{R}^{m} \rightarrow \mathbb{R}^{k} \\
& d \mapsto r^{i}=\left(d_{j}\right)_{j \in \mathbb{I}^{i}},
\end{aligned}
$$

where each $\mathbb{I}^{i}$ denotes the set of indices of $k$ voxels related to each region of interest $r^{i}$. For simplicity, we will assume that these regions do not overlap, i.e., $\forall(i, j) \in$ $\{1, \ldots, q\}^{2}, \mathbb{I}^{i} \cap \mathbb{I}^{j}=\varnothing$ if $i \neq j$.

Following the approach used in the Channelized Hotelling observer (CHO) [10], itself a variant of Hotelling observer (HO) [7], we will opt for channelized regions of interest. The advantage of the channel mechanism is twofold: reducing the complexity in the computation and being able to characterize the human visual system in certain conditions by choosing adequate channels. The channelized regions of interest result from the decomposition of each original region of interest into a set of $c$ discriminative features. The latter ones are represented by a matrix $U \in \mathbb{R}^{k \times c}$ where each column represents a single channel with size equal to the one of the original region of interest, so that the $i$-th channelized region of interest reads $v^{i}=U^{\top} r^{i}$.

We are now ready to formulate our detectability criterion. Consider an index $i \in\{1, \ldots, q\}$, and the associated $v^{i}$. The detection task can be expressed as a binary hypothesis test, defining $\mathcal{H}_{0}$ for microcalcification-free signal and $\mathcal{H}_{1}$ for presence of microcalcification. More precisely, the following two statistical cases have to be distinguished for $r^{i}$ :

$$
\begin{cases}\mathcal{H}_{0}: & r^{i}=b^{i}+\gamma^{i} \\ \mathcal{H}_{1}: & r^{i}=\mu^{i}+b^{i}+\gamma^{i}\end{cases}
$$

where $b^{i}, \mu^{i}$, and $\gamma^{i}$ model the background, the signal of interest (microcalcification) and the noise, respectively. The noise is here assumed to be zero-mean multivariate Gaussian. We propose to define the detectability through the maximization of the log-likelihood ratio under the two aforementioned hypotheses. This one is expressed as

$$
R=\sum_{i=1}^{q} \log \left(\frac{\mathbf{p}\left(v^{i} \mid \mathcal{H}_{1}\right)}{\mathbf{p}\left(v^{i} \mid \mathcal{H}_{0}\right)}\right)
$$

where $\mathrm{p}\left(v^{i} \mid \mathcal{H}_{1}\right)\left(\right.$ resp. $\left.\mathrm{p}\left(v^{i} \mid \mathcal{H}_{0}\right)\right)$ denotes the probability density function of $v^{i}$ in the presence (resp. absence) of microcalcification. In order to compute (6), we need to estimate the sample means and covariance matrices of the channelized regions of interest conditioned to both hypothesis. It is worth noticing that computing the maximum likelihood ratio for a single image is similar to computing the template of Channelized model observer which is obtained by linear disciminant analysis [12]. To do so, we will consider two training datasets $F_{0}$ and $F_{1}$ with the same cardinality $t$ :

$$
\begin{aligned}
& F_{0}=\left\{\tilde{r}_{\mid \mathcal{H}_{0}}^{j} \in \mathbb{R}^{k} \mid j \in\{1, \ldots, t\}\right\} \\
& F_{1}=\left\{\tilde{r}_{\mid \mathcal{H}_{1}}^{j} \in \mathbb{R}^{k} \mid j \in\{1, \ldots, t\}\right\},
\end{aligned}
$$


from which we deduce, for every $j \in\{1, \ldots, t\}$ and $\ell \in$ $\{0,1\}, \tilde{v}_{\mid \mathcal{H}_{\ell}}^{j}=U^{\top} \tilde{r}_{\mid \mathcal{H}_{\ell}}^{j}$. The estimation of the covariance matrix $\Sigma$ of the channelized noise is deduced by

$$
\begin{aligned}
\overline{\tilde{v}_{\mid \mathcal{H}_{\ell}}} & =\frac{1}{t} \sum_{j=1}^{t} \tilde{v}_{\mid \mathcal{H}_{\ell}}^{j}, \quad \ell \in\{0,1\} \\
\Sigma & =\frac{1}{2 t} \sum_{j=1, \ell \in\{0,1\}}^{t}\left(\tilde{v}_{\mid \mathcal{H}_{\ell}}^{j}-\overline{\tilde{v}_{\mid \mathcal{H}_{\ell}}}\right)\left(\tilde{v}_{\mid \mathcal{H}_{\ell}}^{j}-\overline{\tilde{v}_{\mid \mathcal{H}_{\ell}}}\right)^{\top} .
\end{aligned}
$$

Based on these expressions, the log-likelihood ratio in (6) can be re-expressed as

$$
R=\sum_{i=1}^{q} \frac{1}{2}\left(\mu^{i}\right)^{\top} U \Sigma^{-1} U^{\top} \mu^{i}+\left(\mu^{i}\right)^{\top} U \Sigma^{-1} U^{\top} r^{i} .
$$

Since the $\mu^{i}$, s are fixed, the quantity to be maximized is

$$
D(d)=\sum_{i=1}^{q}\left(\mu^{i}\right)^{\top} U \Sigma^{-1} U^{\top} S_{i} d
$$

which yields our penalization term for the detectability of the calcifications in any reconstructed volume $d \in \mathbb{R}_{+}^{m}$.

\section{PRACTICAL IMPLEMENTATION}

The evaluation of the proposed detectability function requires the setting of (i) regions of interest (ROIs) identifying the location of potential calcifications, (ii) the signal of interest $\mu=\sum_{i=1}^{q} S_{i}^{\top} \mu^{i}$ and the associated covariance matrix $\Sigma$. To this aim, a possibility is to resort to Computer-Aided Detection tools [13] that have the ability to locate pathological objects in a medical image. Following classical approaches which are developed to identify lesions based on their size [14], we implement our detectability function to be specifically suited to the detectability of lesions with a size in a specific range. We thus propose a strategy for the construction of signal $\mu$, and the associated regions of interest $\left(\mathbb{I}^{i}\right)_{1 \leq i \leq q}$, based on a TV-based reconstruction of the volume. Note that the covariance matrix $\Sigma$ is learnt using training datasets as described in the previous section.

\subsection{Signal estimation}

In order to build signal $\mu$, we rely on the resolution of the following constrained problem:

$$
\underset{d \in C}{\operatorname{minimize}} \frac{1}{2}\|p-A d\|_{2}^{2}
$$

where $C=\left\{d \in \mathbb{R}_{+}^{m} \mid\|\nabla d\|_{1,2} \leq \eta\right\}$ and $\eta>0$ defines the required bound constraint on the total-variation of the volume, defined as the $\ell_{1,2}$ norm of its discrete gradient $\nabla d$ along the 3 axes. The above objective function is convex, but non differentiable because of the presence of the constraint terms. To solve this minimization problem, we resort to a parallel forward-backward based Primal-Dual approach [15], which presents the advantage of a reduced complexity per iteration (in particular, no inversion of linear operators is required), and benefits from convergence guarantees.

Starting from the resulting regularized TV-based solution $d^{*}$, we propose then to apply morphological operators in order to detect the voxels containing structures suspected to be calcifications whose size lies in a specific range. We opt for an opening operator using a disk as the structuring element that we apply slice per slice. In order to keep structures with radius size in the range $\left[\rho_{\min }, \rho_{\max }\right]$ where $0<\rho_{\min }<\rho_{\max }$, we apply the following steps:

1. Apply opening with radius $\rho_{\min }$ on $d^{*}$ to generate $d_{\mid \rho_{\min }}^{*}$.

2. Apply an opening with radius $\rho_{\max }$ on $d_{\mid \rho_{\min }}^{*}$ to generate $d_{\mid \rho_{\max }}^{*}$.

3. Subtract $d_{\mid \rho_{\min }}^{*}$ from $d_{\mid \rho_{\max }}^{*}$.

The output of the above method leads to our estimated $\mu$ which is expected to contain high voxel intensities in zones where structures are present in the predefined radius range, and very low intensities otherwise.

\subsection{Construction of the ROIs}

The estimated signal $\mu$ we build is not only useful for the detectability function, but also for the construction of the ROIs themselves, as the local maxima of $\mu$ correspond to sought areas for microcalcifications. We propose to extract local maxima from a MaxTree [16] representation computed using 26-connectivity. This approach allows representing the volume in the form of a tree where each node corresponds to a connected component of similar graylevel. The nodes are then connected hierarchically depending on their intensity. By construction, the leaves present the highest graylevel intensities, that are the local maxima. Determining the set of pixels included in each leaf allows us to construct a list of $q$ representative pixels that we define to be the centers of our ROIs. We then deduce the $\left(\mathbb{I}^{i}\right)_{1 \leq i \leq q}$ sets by imposing a given ROI size enabling the inversion of the covariance matrix while minimizing the overlapping of ROIs.

\section{EXPERIMENTAL RESULTS}

We now present experimental results to assess the performance of our method on both physical phantom and clinical data. Each dataset, contains 9 projections obtained using a DBT commercial system (Senographe Essential, GE Healthcare) with an angular range of $25^{\circ}$. The dimension of the detector is $3062 \times 2394$ with $100 \mu^{2}$ detector elements. The images are reconstructed with a $100 \mu \mathrm{m} \times 100 \mu \mathrm{m} \times 1 \mathrm{~mm}$ 
sampling grid. We aim at enhancing the detectability of small microcalcifications with size ranging from $0.2 \mathrm{~mm}$ to $0.4 \mathrm{~mm}$. A detection map is generated as explained in Section 4, with the latter size range. The ROI size is $51 \times 51 \times 5$ pixels. The total number $q$ of ROIs depends on the considered data and varies from $10^{5}$ to $10^{6}$. The covariance matrix $\Sigma$ is learnt from a database of 400 images, with size equal to the ROIs ones, half of them containing lesion shapes while the others represent healthy tissues. These images are synthetic ones generated by using a software available at GE Healthcare [17]. Various types of background models are used, namely uniform background for the phantom data, and textured background for the clinical data. Finally, LaguerreGauss channels [18] are employed for the matrix $U$, whose parameters are tuned depending on background type so as to maximize the area under ROC curve following the $\mathrm{CHO}$ framework [10]. Problem (3) is solved with a smoothed total variation regularization by employing a projected gradient algorithm, initialized with FBP solution, and run until the difference of objective function values between consecutive iterates is less than $10^{-1}$. The regularization parameter and bound $\eta$ associated to the TV term are adjusted based on visual inspection, so as to optimize the global quality of the reconstruction.

\subsection{Physical Phantom Data}

We first use the physical phantom data described by the American College of Radiology (ACR) for measuring the physical standards baseline in mammography. Its dimension is of $45 \times 102 \times 108 \mathrm{~mm}^{3}$. For the sake of visualization, we choose to present only zoomed visual results on a region containing 6 lesions with known locations and size $0.32 \mathrm{~mm}$. Fig. 1 illustrates the influence of a change in the parameter $\alpha$. We observe that the higher the value of $\alpha$, the more visible the microcalcifications. This parameter is expected to control the sensitivity of detection for the microcalcifications in the reconstructed DBT volume. Too high values of this parameter may however have a tendency to spread the size of the lesions. Note that, despite the high numbers of ROIs, the enhancement is only effective in the microcalcifications areas, so displaying the absence of false positive in the detection process.

\subsection{Clinical Data}

We now illustrate a representative case of 9 assessed clinical data, displayed in Fig 2. One can notice that with a suitable value of the weight $\alpha$ for the clinical term, we significantly enhance the visibility of the less obvious microcalcifications. We highlight that the proposed model allows a better visibility of the microcalcifications when compared with a classical reconstruction (i.e., when $\alpha=0$ ) while not degrading the visual homogeneity in the reconstructed image.

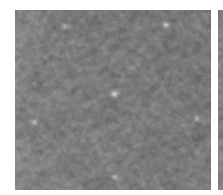

(a) $\alpha=0$

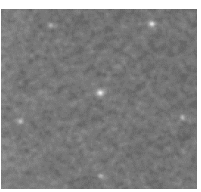

(b) $\alpha=250$

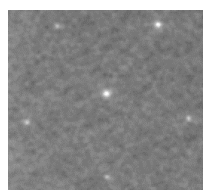

(c) $\alpha=500$

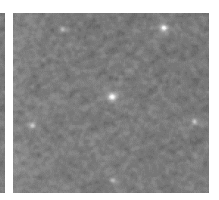

(d) $\alpha=750$
Fig. 1: Region of ACR phantom slice containing 6 ROIs: Enhancement level for different clinical term magnitudes.


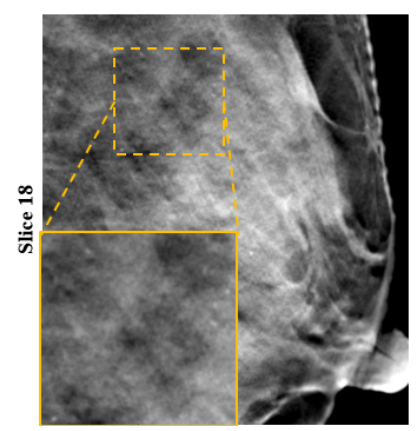

(a) $\alpha=0$

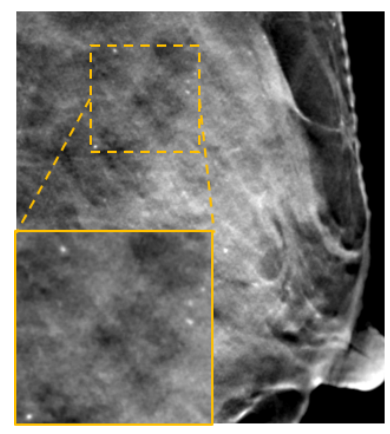

(b) $\alpha=2500$
Fig. 2: Sample of slices of a DBT reconstruction with size $2227 \times$ $594 \times 44$ : (a) DBT reconstruction without the clinical term. (b) DBT reconstruction with the proposed method.

\section{CONCLUSION}

We proposed a new method aimed at enhancing the detectability of microcalcifications in DBT reconstruction as the targeted clinical task. We defined a detectability function, which is computed following an approach similar to the mathematical model observer $\mathrm{CHO}$ and we incorporated it as an a priori term in the proposed optimisation approach. We demonstrated on both synthetic and clinical data the potential interest of our method with respect to standard DBT reconstruction in terms of cancer detection.

In order to improve the recovery of the shapes of the lesions, we plan in our future work to consider more adequate channels in the definition of the proposed detectability function. We intend also to assess microcalcifications detectability performance through psychovisual evaluation sessions. 


\section{REFERENCES}

[1] T. Wu, R.H. Moore, E.A. Rafferty, and D.B. Kopans, "A comparison of reconstruction algorithms for breast tomosynthesis," Medical Physics, vol. 31, no. 9, pp. 2636-2647, 2004.

[2] E.Y. Sidky, I.S. Reiser, R. Nishikawa, and X. Pan, "Image reconstruction in digital breast tomosynthesis by total variation minimization," in Proc. SPIE Medical Imaging, San Diego, CA, March 2007, vol. 6510.

[3] E.Y. Sidky, X. Pan, I.S. Reiser, R.M. Nishikawa, R.H. Moore, and D.B. Kopans, "Enhanced imaging of microcalcifications in digital breast tomosynthesis through improved image-reconstruction algorithms," Medical Physics, vol. 36, no. 11, pp. 4920-4932, 2009.

[4] E. Metin, Y. Isa, K. Mustafa, and A. Aydin, "An iterative tomosynthesis reconstruction using total variation combined with non-local means filtering," BioMedical Engineering OnLine, vol. 13, no. 1, pp. 65, May 2014.

[5] E. L. Piccolomini and E. L. Morotti, "A fast Total Variation-based iterative algorithm for digital breast tomosynthesis image reconstruction," Journal of Algorithms \& Computational Technology, vol. 10, no. 4, pp. 277-289, 2016.

[6] S.D. Rose, A.A. Sanchez, E.Y. Sidky, and X. Pan, "Investigating Simulation-Based Metrics for Characterizing Linear Iterative Reconstruction in Digital Breast Tomosynthesis," Medical Physics, vol. 44, pp. 279-296, September 2017.

[7] H.H. Barrett, J.I.E Yao, J.P. Rollandt, and K.J. Myerst, "Model observers for assessment of image quality," Proceedings of the National Academy of Sciences, vol. 90, no. November, pp. 9758-9765, 1993.

[8] S. Park, J. M. Witten, and K.J. Myers, "Singular vectors of a linear imaging system as efficient channels for the bayesian ideal observer," IEEE Transactions on Medical Imaging, vol. 28.5, pp. 657-668, 2009.

[9] R.L. Van Metter, J. Beutel, and H. L. Kundel, Handbook of Medical Imaging, vol. 1, Laurel, MD, 2000.

[10] L. Platiša, B. Goossens, E. Vansteenkiste, S. Park, B.D. Gallas, A. Badano, and W. Philips, "Channelized Hotelling observers for the assessment of volumetric imaging data sets," Journal of the Optical Society of America A, vol. 28, pp. 1145-1163, 2011.

[11] I. Sechopoulos, "A review of breast tomosynthesis. Part II. Image reconstruction, processing and analysis, and advanced applications," Medical Physics, vol. 40, pp. 014302, 2013.
[12] R.A. Fisher, Statistical Methods for Research Workers, Number V. Genesis Publishing Pvt Ltd, 1925.

[13] I. Christoyianni, A. Koutras, E. Dermatas, and G. Kokkinakis, "Computer aided diagnosis of breast cancer in digitized mammograms," Computerized Medical Imaging and Graphics, vol. 26, no. 5, pp. 309 - 319, 2002.

[14] F.W. Wheeler, A.G.A. Perera, B.E. Claus, S. Muller, G. Peters, and J.P. Kaufhold, "Micro-calcification detection in digital tomosynthesis mammography," in Proc. SPIE Medical Imaging, San Diego, CA, March 2006, vol. 6144 .

[15] N. Komodakis and J.C. Pesquet, "Playing with Duality : An Overview of Recent Primal-Dual Approaches for Solving Large-Scale Optimization Problems," IEEE Signal Processing Magazine, vol. 32, no. 6, pp. 31 - 54, 2014.

[16] C. Berger, T. Geraud, R. Levillain, N. Widynski, A. Baillard, and E. Bertin, "Effective Component Tree Computation with Application to Pattern Recognition in Astronomical Imaging," in Proc. IEEE International Conference on Image Processing (ICIP 2007), San Antonio, TX, 16-19 September 2007, vol. 4, pp. 41-44.

[17] Z. Li, A. Desolneux, S. Muller, P. Milioni de Carvalho, and A.K. Carton, "Comparison of microcalcification detectability in FFDM and DBT using a virtual clinical trial," in Proc. SPIE Medical Imaging, Houston, Texas, February 2018, vol. 10577.

[18] S. Park, H.H. Barrett, E. Clarkson, M.A. Kupinski, and K.J. Myers, "Channelized-ideal observer using Laguerre-Gauss channels in detection tasks involving non-Gaussian distributed lumpy backgrounds and a Gaussian signal," Journal of the Optical Society of America A, vol. 24, no. 12, pp. B136-B150, December 2007. 